\title{
Jefferson Lab Physics Overview: Recent Results
}

\author{
Z.-E. Meziani* \\ Temple University - Department of Physics \\ 1900 N. 13th St., Philadelphia, PA 19122-6082 - USA
}

I review highlights of the Jefferson Lab nucleon structure program. I shall emphasize recent results from experiments exploring the spin structure of the nucleon and from dedicated experiments aimed at accessing the generalized parton distributions (GPDs) [1].

\section{Introduction}

A very rich experimental program on the structure of the nucleon has been carried out at Jefferson Laboratory using the continuous electron beam with a maximum energy of $6 \mathrm{GeV}$ and about $80 \%$ polarization. A series of polarized targets, ${ }^{3} \mathrm{He}$ in $\mathrm{Hall} \mathrm{A}$ and $\mathrm{NH}_{3}$ and $\mathrm{ND}_{3}$ in hall $\mathrm{B}$ and $\mathrm{C}$, combined with a variety of detection schemes, using high resolutions or large acceptance spectrometers, provided for the needed luminosity critical to precision measurements of asymmetries and polarized cross sections in deep inelastic scattering or deep virtual Compton scattering (DVCS) . There is now a large body of spin structure data on the proton, deuteron and neutron that allows us to study quantum chromodynamics (QCD) in the non-perturbative regime, that is at a scale of about $\Lambda_{Q C D}$. Sum rules are used to test our understanding of the theory as we decrease the probe resolution from the size of current quarks to that of the nucleon passing by the size of constituent quarks. Furthermore, a new series of dedicated DVCS experiments have been performed to provide precision data necessary to constrain the generalized parton distributions (GPDs), required for a three-dimensional mapping of the internal nucleon structure.

\section{Sum rules $Q^{2}$ evolution}

Sum rules involving the spin structure of the nucleon offer an important opportunity to study QCD. Among the examples are the Bjorken sum rule[2] at infinite four-momentum transfer $\left(Q^{2}=\infty\right)$ and the Gerasimov, Drell and Hearn (GDH) sum rule[3] at the real photon point $\left(Q^{2}=0\right)$. These sum rules relate the first moments of the spin structure functions (or, equivalently, the spin-dependent total photoabsorption cross sections) to the nucleon's static properties. The above sum rules are based on "unsubtracted" dispersion relations and the optical theorem[4]. Furthermore, another general assumption, such as a low energy theorem[5] for the GDH sum rule and operator production expansion (OPE) [6] for the Bjorken sum rule, is needed to relate the Compton amplitude to a static property. In the case of the GDH sum rule it is the anomalous magnetic moment $\kappa_{p, n}$ while for the Bjorken sum rule it is the nucleon axial coupling constant $g_{A}$. The large set of new spin structure of the nucleon data allows us to address the convergence of the expansion, as $Q^{2}$ decreases form very large values to values of the order of $\Lambda_{Q C D}$, and to evaluate the higher twists contributions.

One example is the study of the evolution to low $Q^{2}$ values of the Bjorken sum in order to extract the higher twists contributions. These contributions contain information about

\footnotetext{
*This work is supported in part by DOE grant contract DE-FG02-94ER40844 .
} 
quark-quark and quark-gluon correlations. The Bjorken sum rule is evaluated at finite $Q^{2}$ using the following expression:

$$
\Gamma_{1}^{p-n}=\int_{0}^{1}\left(g_{1}^{p}-g_{1}^{n}\right) d x=\frac{g_{A}}{6}\left[1-\frac{\alpha_{\mathrm{s}}}{\pi}-3.58\left(\frac{\alpha_{\mathrm{s}}}{\pi}\right)^{2}-20.21\left(\frac{\alpha_{\mathrm{s}}}{\pi}\right)^{3}+\ldots\right]+O\left(\frac{1}{Q^{2}}\right) .
$$

Deviations from the above expression are due to higher twists contributions which can be extracted by direct comparison with the data over a wide range of $Q^{2}$.

Figure 1 shows the evolution of the Bjorken integral when the contribution of elastic scattering is not included. The Bjorken sum rule sets the absolute scale for $\bar{\Gamma}_{1}^{p-n}$ at large $Q^{2}$ and the difference between the leading twist contribution shown by the grey band in Figure 1 and the data gives an estimate of higher twists as $Q^{2}$ decreases to about $1 \mathrm{GeV}^{2}$. An overall suppression of higher twist effects is observed to surprisingly low $Q^{2}$. An analysis of the data, which includes the elastic contribution, down to $Q^{2}=0.8 \mathrm{GeV}^{2}$ allowed the extraction of the non-singlet twist-4 matrix element $f_{2}^{p-n}[7]$. The singlet pieces corresponding to the proton and the neutron were determined in References [8] and [9] respectively. The combination of the twist- $4 f_{2}^{p-n}$ matrix element with the twist-3 matrix element $d_{2}^{p-n}$ extracted from these measurements of $g_{1}$ and $g_{2}$ gave access to "color polarizabilities" for the first time with limited precision. More data from the Eg1b experiment in Hall B with much higher statistical precision will be available soon for publication.

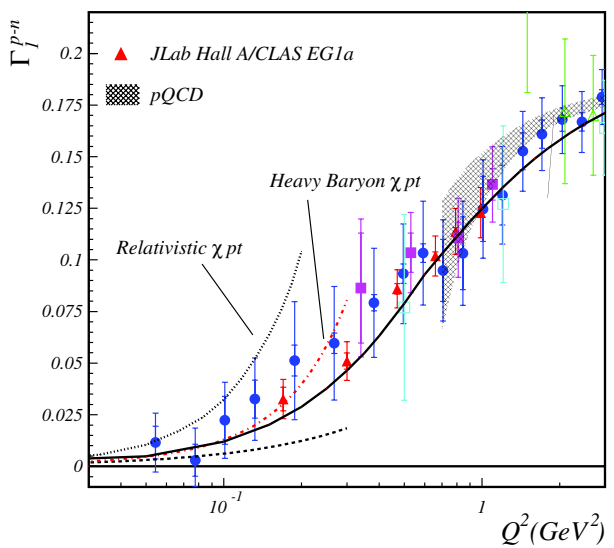

Figure 1: Data on the evolution of the Bjorken integral excluding the elastic contribution which is negligible at large $Q^{2}$ but becomes significant below $Q^{2}=1 \mathrm{GeV}^{2}$. The leading twist pQCD evolution is shown by the grey band. Close to the photon point $\left(Q^{2}=0\right)$ the covariant chiral perturbation and the heavy baryon chiral perturbation calculations are shown.

\section{Helicity Dependent Parton Distributions}

The virtual photon-neutron asymmetry $A_{1}^{n}$ and spin structure function $g_{1}^{n}$ are poorly known in the valence quark region $(x>0.3)$. This shortcoming is due to the small scattering cross sections at large $x$ and $Q^{2}$ combined with a lack of high polarized luminosity facilities. This region, however, is clean and unambiguous since it is not polluted by sea quarks and gluons offering thus a unique opportunity to test predictions that are difficult if not impossible at low $x$.

The set of predictions of $A_{1}^{n}$ in the valence quark region fall into two categories, those of relativistic constituent quark models (RCQM) which break $\mathrm{SU}(6)$ symmetry in the ground state wave function by hyperfine interaction $[10,11,22]$ and include orbital angular momentum implicitly, and those of perturbative quantum chromodynamics (pQCD) with a hadron 
helicity conservation (HHC) constraint $[12,13]$ as $x \rightarrow 1$ which break $\mathrm{SU}(6)$ symmetry dynamically.

The difference between these approaches is dramatic when the constituents flavor-spin decomposition is performed. For a proton and in the case of pQCD with HHC, we have $\Delta u(x) / u(x) \rightarrow 1$ and $\Delta d(x) / d(x) \rightarrow 1$, while for the case of RCQM's $\Delta u / u \rightarrow 1, \Delta d / d \rightarrow$ $-1 / 3$. We note that in leading order pQCD with HHC $\Delta d / d$ changes sign from negative at low $x$ to positive starting at around $x=0.3$.

Using Jefferson Lab unparalleled polarized luminosity, data of the asymmetries of the neutron $A_{1}^{n}[16]$ and the proton $A_{1}^{p}[17]$ were obtained. The quark parton model interpretation of $g_{1}$ and $F_{1}$ was used to perform a flavor decomposition of the spin dependent quark distributions assuming a negligible strange quark contribution above $x=0.3$. The up-quark and down-quark distributions obtained along with results from HERMES semi-inclusive measurements [18] are shown in Fig. 2.

The solid line is a pQCD leading order fit to the world data using the HHC constraint as $x \rightarrow 1$. The long dashed line correspond to an RCQM prediction [22]. It is clear that up to $x=0.6$ the data favor the RCQM rather than the HHC pQCD based calculations. While in the former some OAM is included through the small components of the nucleon wave function in the latter no orbital angular momentum (OAM) until recently [14]. The result of including the

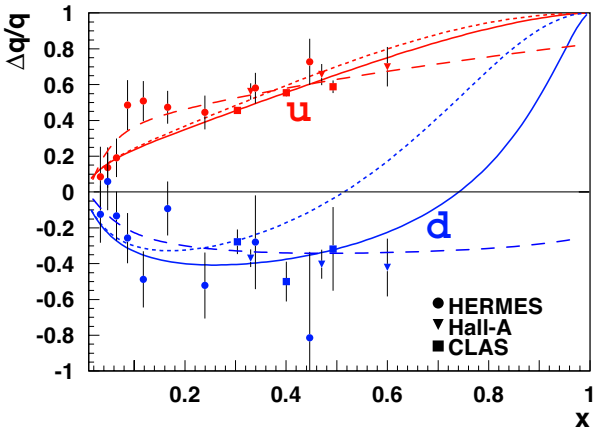

Figure 2: Spin-flavor dependent up-quark and down-quark distributions for a proton extracted from this experiment and the world data using the quark-parton model. The curves describe a PQCD leading order calculation [14] without (short dashed line) and with orbital angular momentum (solid line). The long dashed line is a quark-diquark calculation from Ref. [22] described in the text. OAM is shown in Fig. 2 with the solid line, where the agreement with the data is fair up to $x=0.6$. Of course this gives a strong motivation to test the role of OAM at even larger $x$ than 0.6 , where the difference between $\mathrm{pQCD}$ and the RQCM still remains large.

These results point to the importance of considering the orbital momentum of quarks in the nucleon wave function and in the extraction of the nucleon universal quark-helicity distributions.

\subsection{Quark-hadron duality in the spin structure of the nucleon}

One of the fascinating aspects of nucleon structure, known as "quark-hadron duality", is an observation made in the early 70's by Bloom and Gilman[19] while investigating the spinindependent nucleon response in deep inelastic lepton scattering (DIS). These authors found that, in the scaling regime at large momentum transfers, this response is well described by an average over the resonances structure at lower momentum transfers. Subsequently, in an attempt to explain this observation within the framework of QCD, De Rújula, Georgi and Politzer [20] used the OPE method to suggest a possible link between the average over the resonances response and the DIS scaling response. While many studies were performed 
on the spin-independent response functions of the nucleon [57, 21], a renewed interest has emerged in testing this "duality" behavior in the spin-dependent response functions [22, 23]. In principle with a deeper understanding of QCD and its confinement properties one should be able to predict the observed behavior in either case. With the OPE method one has the opportunity to test the validity of our expansion at low momentum transfers by extracting the higher twist contributions and investigating the convergence and breakdown of such an expansion. Compared to the spin-independent response of the nucleon, the study the spin-dependent response offers a new variety of matrix elements of operators which describe quark-quark and quark-gluon interactions beyond the naive quark-parton model.

We show in Fig. 3 new preliminary results of JLab experiment E01-012 [28] where the virtual photon-nucleus asymmetry $A_{1}$ of ${ }^{3} \mathrm{He}$ was measured at several momentum transfers with excitation energies spanning the nucleon resonance region. For the lowest $Q^{2}$ data points, the prominent feature is the $\Delta$ resonance with a noticeable negative asymmetry. As $Q^{2}$ increases we notice that the asymmetry crosses over to positive values and becomes $Q^{2}$ independent similar to the DIS case. This is suggestive to a behavior of the resonance region similar to that of the DIS region and thus to a duality phenomenon. This behavior is comparable to what was observed in the proton case [23]. This duality has been studied quantitatively using the new data and it is shown that "global duality" of the neutron and ${ }^{3} \mathrm{He}$ polarized structure function $g_{1}$ holds well above $Q^{2}=1.8 \mathrm{GeV}^{2}$.

\section{Color Polarizabilities}

While $g_{1}$ discussed earlier can be under-

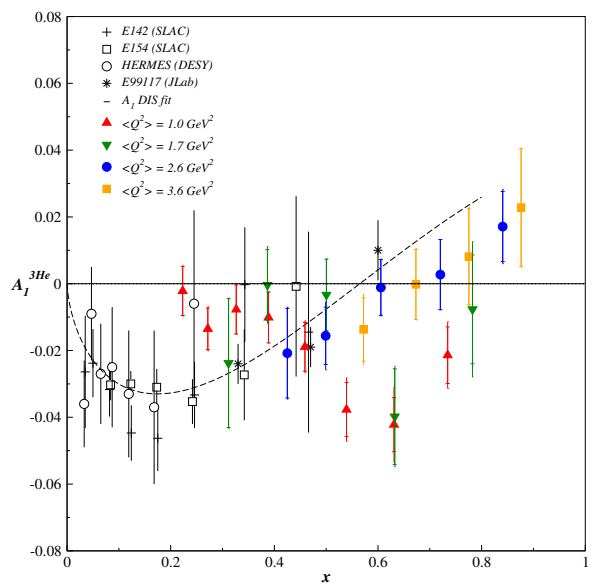

Figure 3: Preliminary result of the asymmetry $A_{1}$ for ${ }^{3} \mathrm{He}$ in the resonance region along the world ${ }^{3}$ He DIS data $[25,26,27,16]$. The outer error bars are total uncertainties while the inner bars represent the statistical part only. The resonance data of the JLab experiment are represented by the filled symbols. The curve is a fit of the $A_{1}^{3} \mathrm{He}$

stood in terms of the Feynman's parton model which describes the scattering in terms of incoherent parton scattering, $g_{2}$ cannot. Using the operator product expansion (OPE) $[29,30]$, it is possible to interpret the $g_{2}$ spin structure function beyond the simple quark-parton model. In fact $g_{2}$ provides a unique opportunity to study the quark-gluon correlations in the nucleon which are otherwise inaccessible. According to the optical theorem, $g_{2}$ is the imaginary part of the spindependent doubly virtual Compton amplitude which involves the $t$-channel helicity exchange +1 . When it is factorized in terms of parton sub-processes, the intermediate partons must carry this helicity exchange. Because of chirality conservation in vector coupling, massless quarks in perturbative processes cannot produce a helicity flip. Nevertheless, in QCD this helicity exchange may occur in the following two ways: first, single quark scattering in which the quark carries one unit of orbital angular momentum through its transverse mo- 
mentum wave function; second, quark scattering with an additional transversely-polarized gluon from the nucleon target. The two mechanisms are combined in such a way to yield a gauge-invariant result. Consequently, $g_{2}$ provides a direct probe of the quark-gluon correlations in the nucleon wave function. In particular the piece of interesting physics is contained in the second moment in $x$ of a linear combination of $g_{1}$ and $g_{2}$,

$$
d_{2}\left(Q^{2}\right)=\int_{0}^{1} x^{2}\left[2 g_{1}\left(x, Q^{2}\right)+3 g_{2}\left(x, Q^{2}\right)\right] d x
$$

This specific combination of $g_{1}$ and $g_{2}$ filters out the free quark scattering interaction exposing the higher twist or quarkgluon interaction. The quantity $d_{2}\left(Q^{2}\right)$ is a twist-three matrix element which is related to a certain quark-gluon correlation, and describes how the gluon field inside the nucleon responds when this latter is polarized. Due to parity conservation, a color magnetic field $\vec{B}$ can be induced along the nucleon polarization (spin direction) while a color electric field $\vec{E}$ in the plane perpendicular to the polarization". In fact $d_{2}$ can be written as $[31,32]$

$$
d_{2}=\left(2 \chi_{B}+\chi_{E}\right) / 3 .
$$

where $\chi_{B}$ and $\chi_{E}$ are the gluon-field polarizabilities defined in the rest frame of the nucleon using the color-singlet operators $O_{B}=\psi^{\dagger} g \vec{B} \psi$ and $O_{E}=\psi^{\dagger} \vec{\alpha} \times g \vec{E} \psi$ :

$$
\chi_{B, E} 2 M^{2} \vec{S}=\left\langle P S\left|O_{B, E}\right| P S\right\rangle .
$$

where $M$ is the nucleon mass and $\vec{S}$ its spin.

Presently $d_{2}^{p}$ and $d_{2}^{n}$ have been evaluated using state of the art computers in the framework of lattice QCD. The proton $d_{2}$ world data have a precision equivalent to that of the present lattice QCD calculation. This situation might change soon with the rapid increase in computers processing

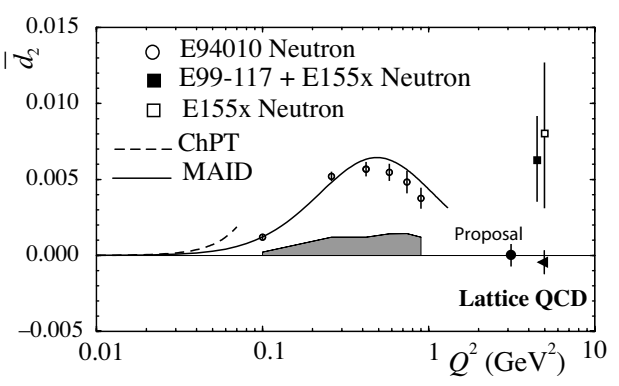

Figure 4: The quantity $\bar{d}_{2}\left(Q^{2}\right)$ is shown at several values of $Q^{2}$. The results of JLab E94-010[39] without the nucleon elastic contribution are the solid circles. The grey band represents their corresponding systematic uncertainty. The SLAC E155 [33] neutron result is the open square. The solid line is the MAID calculation[34] while the dashed line is a $\mathrm{HB} \chi \mathrm{PT}$ calculation [35] valid only at very low $Q^{2}$. The lattice prediction [36] at $Q^{2}=$ $5 \mathrm{GeV}^{2}$ for the neutron $d_{2}$ reduced matrix element is negative but close to zero. We note that many nucleon models not shown in this figure predict a negative or zero value at large $Q^{2}$ where the elastic contribution is negligible. The SLAC datum shows a positive value of $d_{2}^{n}$ but with a rather large error bar. The projected errors of this proposal are the filled circles speed. The neutron data lack the precision required for a meaningful comparison. The present results are very encouraging but much experimental progress needs to be achieved for a definitive comparison with the data.

\subsection{Precision measurement of the neutron $d_{2}$}

Measurements of the helicity dependent cross sections in the large $x$ (valence) region are essential for the determination of higher moments of $g_{1}^{p}$ and $g_{2}^{p}$. These moments are the 
natural connection between experiment and observables calculable in lattice QCD. Lattice QCD calculations do not directly determine spin observables but rather moments of the various polarized and unpolarized structure functions. Lattice QCD collaborations hope to calculate the moments of these structure functions without the quenching approximation and with near-physical pion masses in the next few years employing Teraflop·Year computing resources. Results available today still require extrapolations to the chiral limit[36].

Figure 4 shows how the approved experiment, JLab E06-114 [38], will impact the neutron present comparison between theory and experiment if one uses 20 days of the 6 $\mathrm{GeV}$ polarized electron beam on the polarized ${ }^{3} \mathrm{He}$ target and the Bigbite spectrometer to detect the scattered electrons at large angle $\left(40^{\circ}\right)$. The improvement is rather impressive and will prove to be powerful as both the calculations and the experiment reach new precision levels. This experiment is planned to run in the spring of 2008 ..

\subsection{Spin Asymmetries on the $\mathrm{Nu}-$ cleon Experiment (SANE)}

Although there is a large world data set for $A_{1}^{p}$, the trend of the data in the limit $x \rightarrow 1$ is not clear, and is completely inadequate for estimating all but the first moment of $g_{1}^{p}$. Our goal is to obtain precision $A_{1}^{p}$ and $A_{2}^{p}$ results at the largest possible $x$. A new experiment with a significant increase in Figure of Merit for making high $x$ spin structure function measurements was proposed and approved. The experiment is called SANE (Spin Asymmetries on the Nucleon Experiment) [40], and is based on a 194 msr electron detector viewing the UVa polarized $\mathrm{NH}_{3}$ target operating at $8.5 \times 10^{34}$

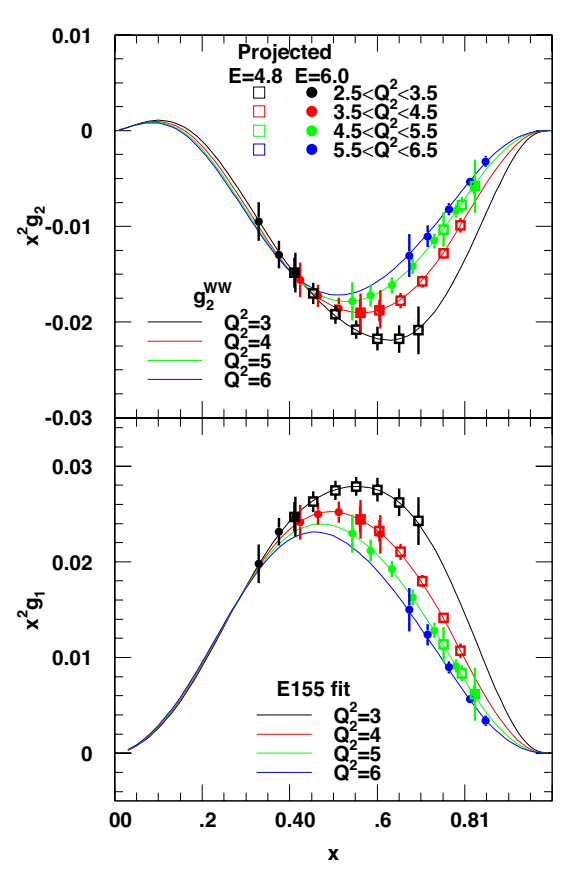

Figure 5: Statistical uncertainties in $x^{2} g_{2}^{p}$ and $x^{2} g_{1}^{p}$ in $\Delta Q^{2}=1 \mathrm{GeV}^{2}$ bins as a function of $x$. The E155 fit [37] to $g_{1} / F_{1}$ was used to calculate $g_{1}$ and $g_{2}^{W W}$ for the solid lines. The projected uncertainties for $6.0 \mathrm{GeV}$ are shown as solid circles and for $4.8 \mathrm{GeV}$ as hollow squares. proton-luminosity.

The proposed measurements of $g_{1}^{p}$ and $g_{2}^{p}$ ( Fig. 5 shows the projected uncertainties of the proposed measurement) will allow us to determine $d_{2}^{p}$ with unprecedented precision at several values of $Q^{2}<6(\mathrm{GeV} / \mathrm{c})^{2}$ for a significantly improved determination of this fundamental quantity.

The SANE experiment is planned to be carried during the summer 2008 in Hall $\mathrm{C}$ using an $\mathrm{NH}_{3}$ polarized target. The scattered electrons will be detected in a newly built Big Electron Telescope Array (BETA) detector. The goal is to perform the measurement of two asymmetries for two different orientations of the target magnetic field relative to the beam direction. And to extract in the proton $A_{1}^{p}$ only limited by systematic errors and a 
simultaneous statistics limited measurement of $g_{2}^{p}$ in the range $0.3 \leq x \leq 0.8$ at an average $Q^{2}=4.5 \mathrm{GeV}^{2}$. The measured $A_{1}$ and $g_{2}$ will be used to study their $Q^{2}$ dependence at fixed $x$ in both the DIS and resonance region, probe the approach of $A_{1}$ to $x=1$ at constant $Q^{2}$ in order to test quark models and pQCD.

Finally, in both the neutron or proton case the sea contribution (or disconnected diagrams) is neglected in the present Lattice QCD calculations. However since $d_{2}$ is a higher moment of spin structure functions those contributions to the total integral are likely to be small. The non-singlet combination $d_{2}^{p}-d_{2}^{n}$ will be used as a true benchmark test of Lattice QCD calculations since this quantity is free from the "disconnected diagrams" (sea contributions) which are less likely to be evaluated in the next few years.

\section{Generalized Parton Distributions}

A comprehensive framework has been developed in the last ten years to unravel the structure of the nucleon [41, 42, 43] (see also reviews and references therein $[44,45$, $46,47])$. In this framework it is shown that the nucleon structure is encoded in the so-called generalized parton distributions (GPDs). For the nucleon, which is a spin $1 / 2$ composite particle, four universal functions for quarks denoted by $H_{f} E_{f}, \tilde{H}_{f}$ and $\tilde{E}_{f}$ describe the helicity-conserving and the helicity-flip nucleon matrix elements of the vector and axial-vector current for quark flavor $q$. Similarly four gluon GPDs describe the gluon structure of the nucleon. Each quark GPD corresponds to the amplitude of probability for removing a quark of momentum fraction $x+\xi$ and restoring it in the nucleon with a momentum fraction

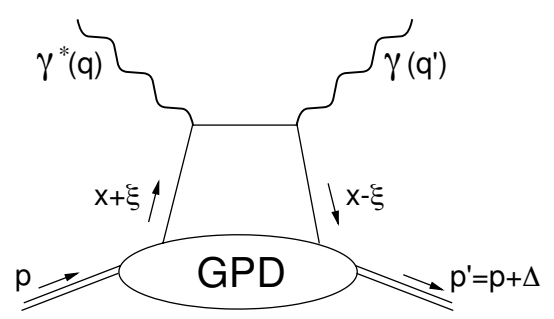

Figure 6: Lowest order amplitude for the virtual Compton scattering process. Shown are the initial four-momentum vectors of the incident virtual photon $q$ and the real scattered photon $q^{\prime}$ as well as the initial $p$ and final $p^{\prime}$ four-momentum of the nucleon. $x$ is the Bjorken scaling variable and $\xi=x /(2-x)$ called skewedness. of $x-\xi$. The overall momentum transfer received by the nucleon is denoted by the Mandelstam variable $t=\Delta^{2}$ and in an impact parameter space, defined by the Fourier transform of the transverse momentum $\Delta_{\perp}$, the GPDs represent distributions of partons of longitudinal momentum $x$ in the transverse plane $[48,49,50,51]$. These functions can be identified, in specific limits of their variables, as the elastic form factors of the nucleon measured in electron elastic scattering or parton distributions measured in inclusive deep inelastic lepton scattering. Within this new framework it was shown that one way to access the GPDs is through deep exclusive processes. However an important aspect of the interpretation of the measured cross section of a given deep exclusive process in terms of GPDs is its factorization into a hard-part that is calculable and a soft-part that embeds the structure, namely the GPDs. 


\subsection{Deep Virtual Compton Scattering}

It was recognized early that Deep Virtual Compton Scattering (DVCS) would be an ideal process to study because factorization might be possible at fixed $x$ and $|t| \ll Q^{2}$ but relatively low $Q^{2}\left(\sim\right.$ few $\left.\mathrm{GeV}^{2}\right)$. This range of $Q^{2}$ is accessible at the present maximum electron beam energy of Jefferson Lab namely $6 \mathrm{GeV}$. The DVCS amplitude is typically expressed in terms of integrals over $x$ of GPDs and has the following form

$$
T^{D V C S} \sim \int_{-1}^{+1} \frac{H(x, \xi, t)}{x \pm \xi-i \epsilon} d x+\ldots \sim \mathcal{P} \int_{-1}^{+1} \frac{H(x, \xi, t)}{x \pm \xi} d x-i \pi H( \pm \xi, \xi, t)
$$

Clearly observables like the cross section of the DVCS process is usually expressed in terms of integrals of GPDs. However, the use of a polarized beam or a polarized target gives access to the imaginary part of the GPDs and thus allows a direct access to the GPDS at specific values of $x$ namely $x= \pm \xi$. This unique situation arises because of the interference between the DVCS and the Bethe-Heitler process through polarization observables.

Up to now most of the existing data showing the DVCS signals originated from non dedicated experiments $[54,55,56]$. Taking advantage of the interference between the DVCS and the Bethe-Heitler process a series of dedicated experiments have been recently performed at Jefferson Lab to determine the DVCS amplitude. In Hall A a dedicated experiment [52] using a highly longitudinally polarized electron beam was scattered of a hydrogen target. The scattered electrons were detected in the standard High Resolution Spectrometer, the outgoing photons and protons were detected in a lead fluoride calorimeter with fast digitizing electronics and and plastic scintillator annular array respectively. This setup allowed for unprecedented luminosities, of

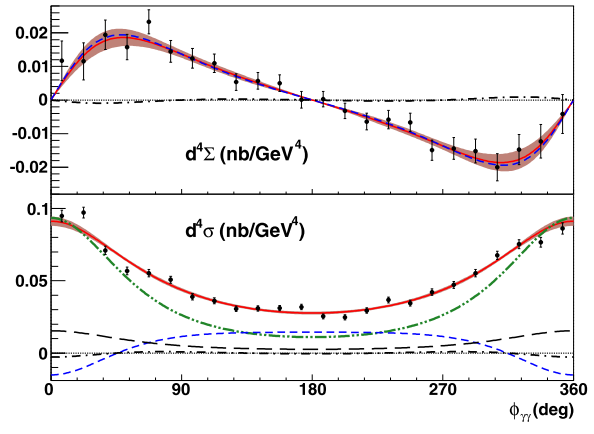

Figure 7: Hall A results for helicity dependent and helicity-independent cross sections as a function of the azimuthal angle $\phi_{\gamma \gamma}$ at $x=0.35, Q^{2}=2.3 \mathrm{GeV}^{2}$ and $t=-0.28 \mathrm{GeV}^{2}$. about $10^{37}$, even with the calorimeter in direct view of the hydrogen target. Helicity dependent cross sections differences $\left(d^{4} \Sigma_{L U}\right.$ were measured at three kinematical points where $x=0.36$ was fixed but $Q^{2}$ increased from $1.5 \mathrm{GeV}^{2}$ to $2.3 \mathrm{GeV}^{2}$. Due to the high resolution and well matched acceptance of the spectrometer and the calorimeter $e p \rightarrow e p \gamma$ events were clearly identified and $e p \rightarrow e \gamma X$ background shape was calibrated using the proton array and subtracted when necessary.

Fig. 7 shows the result of the azimuthal distribution of the helicity dependent and independent DVCS cross section. Although the $Q^{2}$ range scanned is small, no $Q^{2}$ dependence of the extracted amplitudes is observed pointing to scaling and the validity of the dominance of the leading order contribution represented by the diagram of Fig. 6

In a subsequent experiment a deuterium target was used with a slightly different setup to determine the quasi-free DVCS on the neutron. In this case there is direct sensitivity to the GPD $E$ which is needed to evaluate Ji's spin sum rule [57]. Preliminary results can be 
seen in reference [53]

In Hall B another dedicated experiment [58] was performed with full exclusivity of the $e p \rightarrow e p \gamma$ process thanks to the detection of all three final states particles. This was achieved by adding a new inner calorimeter to the standard CLAS configuration and a super-conducting solenoid surrounding the hydrogen target to eliminate the intense Möller scattering background, in the range of $5^{\circ}$ to $15^{\circ}$, which otherwise would swamp the calorimeter at the required luminosity. The calorimeter was made out of lead-tungstate crystals read by state of the art technology developed at the LHC, namely by avalanche photodiode stabilized in temperature. This was the first experiment were this technology has been used successfully. The large acceptance of the spectrometer allowed for a wide kinematic coverage in $x, t$ and $Q^{2}$. The preliminary results of asymmetries were shown at this conference but are not ready for public release.

\section{Conclusion}

A strong and diverse program of nucleon spin structure and of three-dimensional mapping of nucleon internal structure through the determination of GPDs is being carried at Jefferson Lab using the maximum beam energy of $6 \mathrm{GeV}$. This program has a natural extension as the laboratory is preparing for the energy upgrade to $12 \mathrm{GeV}$ incident beam energy. Specific experimental proposals are being submitted to the 12 $\mathrm{GeV}$ program advisory committee and some have already been approved . The reader can obtain more information on $12 \mathrm{GeV}$ proposals at the following urls [59].

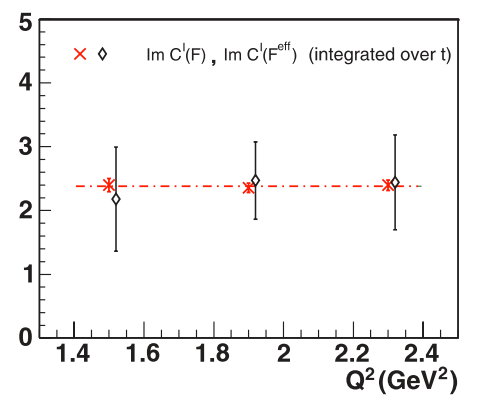

Figure 8: $Q^{2}$ dependence of the imaginary parts of (twist-2) $\mathcal{C}^{\mathcal{I}}(\mathcal{F})$ and (twist-3) $\mathcal{C}^{\mathcal{I}}\left(\mathcal{F}^{\text {eff }}\right)$ angular harmonics averaged over $t$. See Ref. [52] for more details

\section{Acknowledgment}

I would like to first thank the organizers, in particular, A. Caldwell and C. Weiss for the opportunity to present part of the Jefferson Lab exciting program on nucleon structure. I also would like to thank some of my colleagues and collaborators who helped me gather the results presented in this talk, among them J.-P. Chen, M. Garcon, C. Munoz-Camacho, E. Voutier, N. Liyanage, A. Deur, and P. Solvignon. This work was supported by DOE contract DEAC05-84ER40150 under which the Southeastern Universities Research Association (SURA) operates the Thomas Jefferson National Accelerator Facility.

\section{References}

[1] Slides:

http://indico.cern.ch/contributionDisplay. py? contribId=7\&sessionId=2\&conf Id=9499

[2] J. D. Bjorken, Phys. Rev. 148, 1467 (1966); Phys. Rev. D 1, 465 (1970); Phys. Rev. D 1, 1376 (1970).

[3] S. B. Gerasimov, Sov. J. Nucl. Phys. 2, 598 (1965); S. D. Drell and A. C. Hearn, Phys. Rev. Lett. 16, 908 (1966).

[4] see, J. D. Bjorken and S. D. Drell, "Relativistic Quantum Fields", McGraw Hill, New York (1965). 
[5] F. E. Low, Phys. Rev. 96, 1428 (1954).

[6] K. Wilson, Phys. Rev. 179, 1499 (1969).

[7] A. Deur et al., Phys. Rev. Lett. 93, 212001 (2004).

[8] M. Osipenko et al., Phys. Rev. D 71, 054007 (2005), hep-ph/0503018.

[9] Z. E. Meziani et al., Phys. Lett. B 613, 148 (2005), hep-ph/0404066.

[10] F. Close and A. W. Thomas, Phys. Lett. B 212, 227 (1988).

[11] N. Isgur, Phys. Rev. D 59, 034013 (1999).

[12] G. R. Farrar and A. D. Jackson, Phys. Rev. Lett. 35, 1416 (1975).

[13] S. J. Brodsky, M. Burkhardt and I. Schmidt, Nucl. Phys. B 441, 197 (1995).

[14] H. Avakian, S. J. Brodsky, A. Deur and F. Yuan, hep-ph/0705.1553.

[15] I. C. Cloet, W. Bentz and A. W. Thomas, Phys. Lett. B 621, 246 (2005), hep-ph/0504229.

[16] X. Zheng et al. [Jefferson Lab Hall A Collaboration], Phys. Rev. Lett. 92, 012004 (2004) and Phys. Rev. C 70, 065207 (2004)

[17] K. V. Dharmawardane et al. [CLAS Collaboration], Phys. Lett. B 641, 11 (2006), nucl-ex/0605028.

[18] A. Airapetian et al. [HERMES Collaboration], Phys. Rev. Lett. 94, 012002 (2005)

[19] E. D. Bloom and F. J. Gilman, Phys. Rev. Lett. 25, 1140 (1970) and Phys. Rev. D 4, 2901 (1971).

[20] A. De Rújula, H. Georgi and H. D. Politzer, Annals Phys. (N.Y.) 103, 315 (1977).

[21] W. Melnitchouk, R. Ent and C. Keppel, Phys. Rept. 406, 127 (2005), hep-ph/0501217.

[22] F. Close and W. Melnitchouk, Phys. Rev. C 68, 035210 (2003), hep-ph/0302013.

[23] A. Airapetian et al. [HERMES Collaboration], Phys. Rev. Lett. 90, 092002 (2003), hep-ex/0209018.

[24] X. Ji and W. Melnitchouk, Phys. Rev. D 56, 1 (1997).

[25] P. L. Anthony et al. [SLAC E142 Collaboration], Phys. Rev. D 54, 6620 (1996), hep-ex/9610007.

[26] K. Abe et al. [SLAC E154 Collaboration], Phys. Rev. Lett. 79, 26 (1997), hep-ex/9705012.

[27] K. Ackerstaff et al. [HERMES Collaboration], Phys. Lett. B 464, 123 (1999), hep-ex/9906035.

[28] JLab experiment E01-012, N. Liyanage, S. Choi and J.-P. Chen, spokespersons and P. Solvignon, Ph.D. thesis, Temple University (2006).

[29] E. Shuryak and A. Vainshtein, Nuc. Phys. B 201, 141 (1982).

[30] R. L. Jaffe and X. Ji, Phys. Rev. D 43, 724 (1991).

[31] E. Stein et al., Phys. Lett. B 353, 107 (1995).

[32] B. W. Filippone and X. Ji, Adv. Nucl. Phys. 26, 1 (2001).

[33] SLAC E155: P. L. Anthony et al., Phys. Lett. B 553, 18 (2003).

[34] D. Drechsel, S. Kamalov and L. Tiator, Phys. Rev. D63, 114010 (2001).

[35] C. W. Kao, T. Spitzenberg and M. Vanderhaeghen, Phys. Rev. D 67, 016001 (2003).

[36] M. Gockeler et al., Phys. Rev. D 72, 054507 (2005), hep-lat/0506017.

[37] P L. Anthony et al., Phys. Lett. B 493, 19 (2000).

[38] Jefferson Lab experiment E06-114, S. Choi, X. Jiang, Z.-E. Meziani and B. Sawatzky spokespersons.

[39] M. Amarian et al. [Jefferson Lab E94-010 Collaboration], Phys. Rev. Lett. 92, 022301 (2004).

[40] Jefferson Lab experiment E03-110, O. Rondon, S. Choi and Z.-E. Meziani spokespersons.

[41] D. Muller, D. Robaschik, B. Geyer, F. M Dittes and J. Horejsi, Fortsch. Phys. 42, 101 (1994).

[42] A. V. Radyushkin, Phys. Rev. D 56, 5524 (1997).

[43] X. Ji, Phys. Rev. Lett. 78, 610 (1997).

[44] M. Diehl, Phys. Rep. 388, 41 (2003).

[45] X. Ji, Ann. Rev. Nucl. Part. Sci. 54, 413 (2004).

[46] P. Kroll, Prog. Part. Nucl. Phys. 55, 281 (2005).

[47] A. V. Belitskyand and A. V. Radyushki, Phys. Rep. 418, 1 (2005).

[48] M. Burkardt, Phys. Rev. D 62, 071503 (2000).

[49] J. P. Ralston and B. Pire, Phys. Rev. D 66, 111501 (2002).

[50] M. Diehl, Eur. Phys. J. C 25, 223 (2002).

[51] A. V. Belitsky and D. Mueller, Nucl. Phys. A 711, 118 (2002). 
[52] C. Munoz Camacho et al. [Jefferson Lab Hall A Collaboration], Phys. Rev. Lett. 97, 262002 (2006), nucl-ex/0607029.

[53] M. Mazouz, Nucl. Phys. A 782, 41 (2007).

[54] A. Airapetian et al. [HERMES collaboration], Phys. Rev. Lett. 87, 1820012001 and Phys. Rev. D 75 011103 (2007).

[55] S. Stepanyan et al. [CLAS collaboration], Phys. Rev. Lett. 87, 182002 (2001).

[56] S. Chen et al. [CLAS Collaboration], Phys. Rev. Lett. 97, 072002 (2006), hep-ex/0605012.

[57] X. Ji, Phys. Rev. Lett. 78, 610 (1997).

[58] M. Garçon, AIP Conf. Proc. 870, 93 (2006).

[59] http://www.jlab.org/Hall-B/clas12/Physics/ http://www.jlab.org/Hall-C/upgrade/index.html 